\title{
Acute Periprosthetic Hip Joint Infection Caused by Multidrug-Resistant Acinetobacter Baumannii: Is Debridement, Antibiotics, Irrigation, and Implant Retention a Viable Treatment Option?
}

Angelo V. Vasiliadis ${ }^{1}$, Frideriki Poutoglidou ${ }^{2}$, Vasiliki Chatziravdeli ${ }^{1}$, Dimitrios Metaxiotis ${ }^{3}$, Anastasios Beletsiotis ${ }^{1}$

1. Orthopaedics, 2nd Orthopaedic Department, General Hospital of Thessaloniki "Papageorgiou”, Thessaloniki, GRC 2. Department of Anatomy and Surgical Anatomy, School of Medicine, Faculty of Health Sciences, Aristotle University of Thessaloniki, Thessaloniki, GRC 3. Orthopaedics, General Hospital of Thessaloniki "Papageorgiou", Thessaloniki, GRC

Corresponding author: Angelo V. Vasiliadis, vasiliadis.av@gmail.com

\begin{abstract}
In this study, we aimed to investigate the effectiveness of debridement, antibiotics, irrigation, and implant retention (DAIR) in periprosthetic hip joint infection caused by multidrug-resistant (MDR) Acinetobacter baumannii (A. baumannii).
\end{abstract}

From July 2019 to June 2020, we retrospectively reviewed all patients treated for periprosthetic hip joint infections caused by MDR A. baumannii at our institution. The diagnosis of periprosthetic joint infection (PJI) was established based on the Musculoskeletal Infection Society (MSIS) 2018 criteria. The Charlson Comorbidity Index (CCI) was used to estimate the risk of mortality. The patients were followed up for over a year, until their death, or loss to follow-up.

Four patients (three females and one male), with a mean age of 68 years, were included in the study. $A$. baumannii exhibited resistance to fluoroquinolones in all cases. All patients were treated with the DAIR procedure followed by intravenous tigecycline and colistin combination treatment. Prosthesis retention with good functional results was achieved in two patients. One patient required resection arthroplasty and one patient died two months after the initial surgical treatment, yielding a success rate of $50 \%$ for the DAIR procedure.

Periprosthetic hip joint infection caused by MDR A. baumannii is one of the most demanding and challenging complications in orthopaedic practice. This case series suggests that the outcome of the DAIR is affected by a number of factors that are in a complex interplay. Our results indicate a limited success rate for the DAIR procedure in the treatment of a periprosthetic hip joint infection caused by MDR A. baumannii.

Review began 01/25/2021 Review ended 01/30/2021 Published 02/03/2021

\section{() Copyright 2021}

Vasiliadis et al. This is an open access article distributed under the terms of the Creative Commons Attribution License CC-BY 4.0., which permits unrestricted use, distribution, and reproduction in any medium, provided the original author and source are credited.
Categories: Infectious Disease, Orthopedics

Keywords: periprosthetic joint infection, acinetobacter baumannii, dair, arthroplasty, antibiotic treatment

\section{Introduction}

Periprosthetic joint infection (PJI) is a rare but major complication after hip replacement, with an incidence of $0.5-3 \%$; it results in significant morbidity and mortality [1]. The risk of developing a PJI is associated with several potentially patient-related factors, such as sociodemographic characteristics, body mass index (BMI), and medical/surgical history [2]. Management of a PJI is challenging and usually requires complex treatment strategies in order to provide favorable outcomes related to patients' quality of life [3]. For early postoperative PIIs (occurring within 30 days of implantation), debridement, antibiotics, irrigation, and implant retention (DAIR) is the conventional treatment option. The DAIR procedure should be performed promptly after the onset of the symptoms of infection [2]. Failure of the DAIR is related to existing preoperative parameters, such as the immunocompromised state in patients, persistent wound drainage, and specific bacterial pathogens isolated from the culture [4].

\footnotetext{
Acinetobacter species are aerobe gram-negative microorganisms that typically colonize the skin surface. Previous studies report a colonization rate of $25-40 \%$ and $75 \%$ among healthy individuals and hospitalized patients respectively. Moreover, the respiratory tract of patients is frequently colonized by Acinetobacter species during hospitalization [5]. Nosocomial infections caused by Acinetobacter species represent a significant clinical problem, with the overall incidence of PJI due to Acinetobacter species ranging from $0.6 \%$ to $1.2 \%$ according to recent literature [6,7]. Infections due to Acinetobacter Baumannii (A. baumannii) is indicative of severe illness and is related to a $30 \%$ higher risk of mortality [8]. In the present study, we retrospectively reviewed four cases of multidrug-resistant (MDR) A. baumannii PJI, in order to assess the efficacy of the DAIR procedure.
} 


\section{Case Presentation}

We performed a single-center, retrospective case series study. The study was examined and approved by our hospital's Institutional Review Board. Over a period of 12 months (from July 2019 to June 2020), patients over 18 years of age, with a periprosthetic hip joint infection caused by A. baumannii, and who underwent DAIR, were included in the study. Patient data were acquired from the department's medical and surgical indices, as well as the microbiology database. A dedicated data collection form was developed for recording all relevant details. Patients were followed up for over a year, until their death, or loss to follow-up.

The diagnosis of PJI was established based on the Musculoskeletal Infection Society (MSIS) 2018 criteria and the International Consensus Meeting [9]. Therefore, the infection was defined as the presence of a sinus tract communicating with the articular cavity, or the isolation of the pathogen by intraoperative culture in two separate specimens obtained from the affected prosthetic joint, or the presence of at least three of the following minor criteria: i) elevated C-reactive protein (CRP) and erythrocyte sedimentation rate (ESR), ii) elevated synovial fluid white blood cell (WBC) or ++ change on leukocyte esterase test strip, iii) elevated synovial fluid polymorphonuclear neutrophil percentage (PMN\%), iv) a positive histological analysis of the periprosthetic tissue, and v) a single positive synovial culture.

As previously described, PJI was defined as acute when symptom duration was less than three weeks or if the symptoms developed within the first four weeks after arthroplasty [3]. Meanwhile, the Charlson Comorbidity Index (CCI) adjusted for age was calculated. The CCI, an indicator of comorbidity in orthopaedic patients, is commonly used to predict prolonged hospitalization, complications, and mortality. Finally, patients were classified as having a good outcome or treatment failure. Treatment failure was defined based on patients meeting at least one of the following criteria: i) recurrence of the PJI due to A. baumannii strain or a different microorganism, ii) death due to a prosthesis-related infection, iii) clinical, laboratory, or radiological findings suggestive of a PJI at any time after the initial treatment, and iv) a need for resection arthroplasty.

Four patients (three females and one male with a mean age of 68 years, range: 64-74 years) were diagnosed with MDR A. baumannii PJI. Patients' demographics data are presented in Table 1. One patient had a cemented bipolar hemiarthroplasty due to a previous femoral neck fracture, two patients had undergone primary total hip arthroplasty, and one patient had undergone revision total hip arthroplasty. Major comorbidities present were hypertension $(n=3)$, hypothyroidism $(n=2)$, atrial fibrillation $(n=2)$, diabetes mellitus ( $n=1)$, and dyslipidemia $(n=1)$. Main symptoms were pain $(n=4)$, wound drainage $(n=3)$, and fever (temperature of $>37.5-39^{\circ} \mathrm{C}, \mathrm{n}=2$ ). The mean CCI was 51 (range: $21-77$ ) (Table 1). 


\section{Cureus}

\begin{tabular}{|c|c|c|c|c|}
\hline Characteristics & Case 1 & Case 2 & Case 3 & Case 4 \\
\hline Gender & Female & Female & Female & Male \\
\hline Age, years & 67 & 64 & 74 & 67 \\
\hline BMI, $\mathrm{kg} / \mathrm{m}^{2}$ & 22.03 & 20.95 & 25.7 & 30.9 \\
\hline $\mathrm{CCl}, \%$ & 77 & 53 & 21 & 53 \\
\hline Indication & Aseptic loosening & Femoral neck fracture & Femoral neck fracture & Secondary OA \\
\hline Arthroplasty & Revision THA & Primary THA & Bipolar HA & Primary THA \\
\hline Comorbidities & HTN, DLP & $\begin{array}{l}\text { HTN, HT, DM, COPD, anemia, } \\
\text { depression }\end{array}$ & HTN, HT, AF, GERD & AF \\
\hline \multicolumn{5}{|l|}{$\begin{array}{l}\text { Signs and } \\
\text { symptoms }\end{array}$} \\
\hline Pain & Yes & Yes & Yes & Yes \\
\hline Fever & No & Yes & No & Yes \\
\hline $\begin{array}{l}\text { Wound } \\
\text { drainage }\end{array}$ & Yes & Yes & Yes & No \\
\hline \multicolumn{5}{|l|}{ Laboratory findings } \\
\hline WBC, $\times 10^{3} / \mu \mathrm{L}$ & 7.46 & 6.22 & 7.23 & 6.3 \\
\hline CRP, mg/dL & 2.96 & 23.8 & 3.08 & 16.3 \\
\hline ESR, mm/h & 95 & 123 & 72 & 120 \\
\hline $\begin{array}{l}\text { Culture sample } \\
\text { (number) }\end{array}$ & $\begin{array}{l}\text { synovial fluid, deep } \\
\text { tissue (5) }\end{array}$ & $\begin{array}{l}\text { synovial fluid, deep tissue, implant } \\
\text { (5) }\end{array}$ & $\begin{array}{l}\text { Synovial fluid, deep } \\
\text { tissue (5) }\end{array}$ & $\begin{array}{l}\text { Deep tissue, } \\
\text { implant (5) }\end{array}$ \\
\hline IA-DAIR, days & 24 & 20 & 33 & 25 \\
\hline Antibiotics IV (days) & $\mathrm{T}, \mathrm{Co}, \mathrm{V}, \mathrm{Te}(23)$ & T, Co, V (20) & T, Co, V (23) & $\mathrm{T}, \mathrm{Co}, \mathrm{V}, \mathrm{Te}(28)$ \\
\hline $\begin{array}{l}\text { Surgical } \\
\text { management }\end{array}$ & DAIR & DAIR (x 2), Res. A & DAIR & DAIR, Res. A \\
\hline Failed treatment & No & Yes & No & Yes \\
\hline Functional outcome & Independent life & Wheelchair user & Independent life & Deceased \\
\hline Follow-up, months & 19 & 18 & 16 & 2 \\
\hline
\end{tabular}

\section{TABLE 1: Characteristics of four patients with periprosthetic hip joint infection caused by}

multidrug-resistant Acinetobacter Baumannii

The detection limit of CRP was $>0.8 \mathrm{mg} / \mathrm{dL}$

BMI: body mass index; CCl: Charlson Comorbidity Index; OA: osteoarthritis; THA: total hip arthroplasty; HA: hip arthroplasty; HTN: hypertension; DLP: dyslipidemia; HT: hypothyroidism; DM: diabetes mellitus; COPD: chronic obstructive pulmonary; AF: atrial fibrillation; GERD: gastroesophageal reflux disease; T: tigecycline; Co: colistin; V: vancomycin; Te: teicoplanin; Res. A: resection arthroplasty

Mean WBC, ESR, and CRP values at presentation were $6.8 \times 10^{3} / \mu \mathrm{L}$ (range: $6.22 \times 10^{3} / \mu \mathrm{L}-7.46 \times 10^{3} / \mu \mathrm{L}$ ), $102.5 \mathrm{~mm} / 1^{\text {st }}$ hour (range: $72-123 \mathrm{~mm} / 1^{\text {st }}$ hour), and $11.5 \mathrm{mg} / \mathrm{dL}$ (range: $2.96-23.8 \mathrm{mg} / \mathrm{dL}$ ) respectively (normal range - WBC: $4-11 \times 10^{3} / \mu \mathrm{L}$; ESR: $0-20 \mathrm{~mm} / 1^{\text {st }}$ hour; CRP: $<0.8 \mathrm{mg} / \mathrm{dL}$ ). The mean duration from arthroplasty to PJI diagnosis was 18.5 days (range: $12-25$ days). A. baumannii PJI was isolated from culture samples collected from joint synovial fluid, deep tissue cultures, and/or the implant itself (Table 1).

All patients included in the study were treated with the DAIR procedure. Surgical debridement was performed through the previous incision and included thorough irrigation of the joint cavity, sample collection for microbiological cultures (at least five) using separate sterile instruments, and modular 
exchange. The wound and the joint cavity were pulse lavage-irrigated using 6 liters of normal saline. Vancomycin ( $1 \mathrm{~g}$ intravenous every 12 hours) was the initial empirical antibiotic therapy, guided by our local prevalence of pathogens and their drug susceptibility patterns. All cultures were positive for A. baumannii. The antibacterial resistance of $A$. baumannii strains was determined for 17 antibiotics (Figure 1). A. baumannii strains displayed very high minimum inhibitory concentration (MIC) values for at least three classes of antibacterial agents, all penicillins-cephalosporins, fluoroquinolones, and aminoglycosides, thereby defining A. baumannii as MDR [10]. Thereafter, tigecycline (100 mg every 12 hours) and colistin (initial dose of 9 million IU followed by 4.5 million IU every 12 hours) were administrated intravenously for two to four weeks and subsequently switched to oral antibiotic therapy for at least three months. Antibiotic treatment was discontinued once clinical resolution and normalization of serum inflammatory markers were achieved. Laboratory monitoring (CRP, ESR, WBC counts, serum creatinine, and liver enzymes) was performed to assess the effectiveness of the antibiotic therapy and to detect any adverse events or potential toxicity.

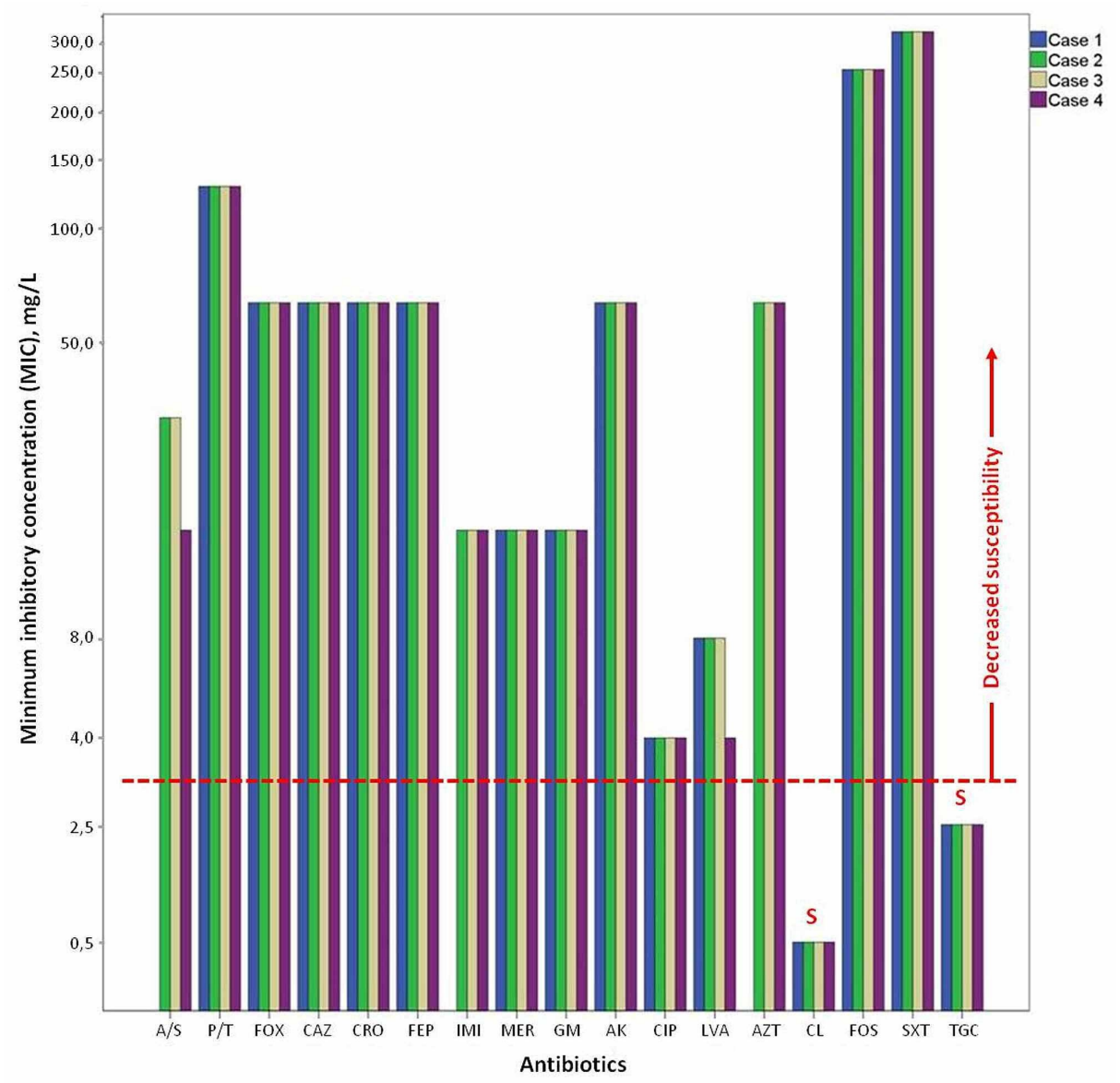

FIGURE 1: Antibacterial susceptibility expressed as a minimum inhibitory concentration (MIC), mg/L, of Acinetobacter baumannii to 17 antibiotics

A/S: ampicillin/sulbactam; P/T: piperacillin/tazobactam; FOX: cefoxitin; CAZ: ceftazidime; CRO: ceftriaxone; FEP: cefepime; IMI: imipenem; MER: meropenem; GM: gentamicin; AK: amikacin; CIP: ciprofloxacin; LVA: levofloxacin; AZT: aztreonam; CL: colistin; FOS: fosfomycin; SXT: trimethoprim/sulfamethoxazole; TGC: tigecycline; S: sensitive

The mean interval between arthroplasty and debridement was 25.5 days (range: 20-33 days). The mean intravenous antibiotic duration was 23.5 days (range: $20-28$ days). Treatment was unsuccessful in two patients; one patient had a recurrence of the infection that required a second debridement and resection arthroplasty. Patient number 4 underwent DAIR, a resection arthroplasty, and died approximately two months after initial surgical treatment. The average duration of the follow-up for the three patients who survived was 17.66 months (range: 16-19 months) (Table 1).

\section{Discussion}

Our approach to treating MDR A. baumannii PJI with the DAIR procedure was only partially successful in the 
present study since the infection was eradicated in only two out of four patients included. The genus Acinetobacter (originating from the Greek word "akinetos", which means non-motile), which was first described in 1911, comprises aerobic gram-negative bacteria that can cause severe wound infections. An increasing number of infections caused by this microorganism has been recorded over the last three decades. Meanwhile, there has been a dramatic increase of antibiotic-resistant strains of the pathogen, limiting antibiotic treatment options for infection and posing a challenge to clinicians [11].

A. baumannii is an opportunistic nosocomial pathogen, rare in orthopaedic practice, which is associated with serious infections with high rates of morbidity and mortality. PJI caused by Acinetobacter species is relatively rare, with an incidence ranging from $0.6 \%$ to $1.2 \%$ in the literature. Surprisingly, in our department, we encountered four cases of $A$. baumannii PJI within a one-year period. The high proportion of PJIs due to A. baumannii, compared to other pathogens, in our study contrasts with the data from other large epidemiological studies [6,7,11]. This could be attributed to contamination by A. baumannii of the surfaces in hospital rooms, such as beds, tables, sinks, medical devices, floor, and walls. Indeed, following the cleaning and disinfection of our medical wards, once the fourth case described in the present study was confirmed, the cases of A. baumannii PJIs were reduced to zero the following year. Contaminated environmental surfaces in hospital rooms can contribute to the transmission of hospital pathogens, including methicillinresistant Staphylococcus aureus, vancomycin-resistant Enterococcus species, Clostridium difficile, Acinetobacter species, and norovirus [12]. Educational and training programs for healthcare workers and the development of new protocols and checklists regarding hand hygiene and surface disinfection could reduce environmental contamination and thereby the rate of hospital-acquired infections.

PJI is essentially a surgical disease and the majority of the cases require some form of surgical intervention. The DAIR remains a key element in the management of PJI, especially when the usual pathogens are involved, such as Enterococcus, Staphylococcus, and Streptococcus [13]. For acute PJIs caused by gram-negative bacteria and managed with the DAIR procedure, the prognosis is relatively good. MartínezPastor et al. [14] report a success rate of $74.5 \%$ for the treatment of gram-negative infections with debridement, implant retention, and antibiotic treatment. Interestingly, they found a positive correlation between favorable outcomes and CRP concentration below $15 \mathrm{mg} / \mathrm{dl}$ at the time of diagnosis and treatment with fluoroquinolones. In our study, we believe that the outcome of DAIR was affected by a number of factors, such as the advanced age of the patients, the comorbidities, the time interval between arthroplasty and DAIR, and the presence of the specific bacterial pathogen, which were in a complex interplay.

Currently, there is no consensus regarding the real efficacy of the DAIR procedure. Previous studies have shown variable treatment success rates ranging from $31 \%$ to $100 \%$ for acute infections. Many factors may have contributed to this wide range of reported success rates. The type of infection, the number and species of pathogens isolated, the time of the initiation of treatment, and patient characteristics are of great importance for a successful clinical outcome [4]. It is reported that joint infection caused by MDR Acinetobacter is associated with higher failure rates and, hence, prosthesis retention is not recommended. The production of a mature biofilm, which evades the host defense, causes a delay in the clinical presentation and leads to failure of the antibiotic treatment due to the lack of a systemic response and is one of the main reasons proposed for DAIR failure [3].

In a recent study, Kazimoglou et al. [15] reported a limited success rate (41\%) for the DAIR procedure for the treatment of seven patients with early-onset hemiarthroplasty infections caused by either gram-positive or gram-negative bacteria. They also found that successful outcomes are positively correlated with a duration of the infection after implantation under two weeks and a sedimentation value lower than $60 \mathrm{~mm} / \mathrm{h}$. However, It should be noted that, in their study, modular components of the prosthesis were not exchanged, which could be the reason for the poor outcomes. On the other hand, Sukeik et al. [16] advocate aggressive irrigation and exchange of modular parts for both early and late infections with a short duration of symptoms, and they achieved a $100 \%$ success rate.

The DAIR procedure may not be a viable treatment option once a mature biofilm is formed around the prosthesis. The DAIR is not recommended if the interval between the onset of the infection and the debridement is more than four weeks. Complete removal of the prosthesis is necessary for patients with a longer duration of symptoms and when a mature biofilm is formed around the implant $[3,11]$. The establishment of a biofilm around a prosthesis contributes to the extensive dissemination of MDR strains of gram-negative bacteria. A. baumannii, a gram-negative microorganism, is capable of forming a biofilm, which serves as a physical barrier to the diffusion of antibacterial agents, rendering it even more resistant $[15,17]$.

Previous studies suggest excellent treatment outcomes for PJIs caused by gram-negative bacteria, susceptible to fluoroquinolones [17,18]. Fluoroquinolones demonstrate not only a favorable pharmacokinetic profile but also an excellent penetration in gram-negative biofilms. However, the increasing fluoroquinolone-resistance rates among gram-negative bacteria and the emergence of MDR Acinetobacter infections have limited their use in PJIs. In our study, the isolated A. Baumannii was resistant to the commonly recommended antibiotics for PJIs in all cases. A combination therapy involving tigecycline and colistin has been shown to decrease bacterial counts, prevent bacterial regrowth at subinhibitory concentrations, and lead to promising results in the treatment of complex PJIs [19,20]. Vila et al. [19], in a 
cases series of three patients with Acinetobacter PJI who were treated with the DAIR and a combination of high-dose tigecycline and colistin in the immediate postoperative period, reported a 100\% success rate, with none of the patients showing any sign of relapse in the follow-up period.

\section{Conclusions}

In conclusion, this study presented a case series of PJI due to MDR A. baumannii managed with the DAIR procedure. Our results show a $50 \%$ success rate in fluoroquinolone-resistant, gram-negative bacteria treated with debridement, implant retention, and a combination of tigecycline and colistin antibiotic therapy. The DAIR procedure resulted in a limited success rate for the management of MDR A. baumannii PJIs. MDR A. baumannii PJI is more challenging to be eradicated compared to infections caused by common gram-positive pathogens. Further long-term studies, with a larger number of patients, are required to validate our results.

\section{Additional Information}

\section{Disclosures}

Human subjects: Consent was obtained or waived by all participants in this study. General Hospital of Thessaloniki IRB issued approval 2021. The study was examined and approved by the General Hospital of Thessaloniki's Institutional Review Board. Conflicts of interest: In compliance with the ICMJE uniform disclosure form, all authors declare the following: Payment/services info: All authors have declared that no financial support was received from any organization for the submitted work. Financial relationships: All authors have declared that they have no financial relationships at present or within the previous three years with any organizations that might have an interest in the submitted work. Other relationships: All authors have declared that there are no other relationships or activities that could appear to have influenced the submitted work.

\section{References}

1. Ahmed SS, Haddad FS: Prosthetic joint infection. Bone Joint Res. 2019, 8:570-2. 10.1302/20463758.812.BJR-2019-0340

2. Kunutsor SK, Whitehouse MR, Blom AW, Beswick AD; INFORM Team: Patient-related risk factors for periprosthetic joint infection after total joint arthroplasty: a systematic review and meta-analysis. PLoS One. 2016, 11:e0150866. 10.1371/journal.pone.0150866

3. Izakovicova P, Borens O, Trampuz A: Periprosthetic joint infection: current concepts and outlook. EFORT Open Rev. 2019, 4:482-94. 10.1302/2058-5241.4.180092

4. Qasim SN, Swann A, Ashford R: The DAIR (debridement, antibiotics and implant retention) procedure for infected total knee replacement - a literature review. SICOT J. 2017, 3:2. 10.1051/sicotj/2016038

5. Seifert H, Dijkshoorn L, Gerner-Smidt P, Pelzer N, Tjernberg I, Vaneechoutte M: Distribution of Acinetobacter species on human skin: comparison of phenotypic and genotypic identification methods. J Clin Microbiol. 1997, 35:2819-25. 10.1128/JCM.35.11.2819-2825.1997

6. Benito N, Mur I, Ribera A, et al.: The different microbial etiology of prosthetic joint infections according to route of acquisition and time after prosthesis implantation, including the role of multidrug-resistant organisms. J Clin Med. 2019, 8:673. 10.3390/jcm8050673

7. Benito N, Franco M, Ribera A, et al.: Time trends in the aetiology of prosthetic joint infections: a multicentre cohort study. Clin Microbiol Infect. 2016, 22:732. 10.1016/j.cmi.2016.05.004

8. Hischebeth GT, Wimmer MD, Molitor E, Seifert H, Gravius S, Bekeredjian-Ding I: Multidrug resistant Acinetobacter baumannii reaches a new frontier: prosthetic hip joint infection. Infection. 2015, 43:95-7. 10.1007/s15010-014-0661-x

9. Parvizi J, Tan TL, Goswami K, Higuera C, Della Valle C, Chen AF, Shohat N: The 2018 definition of periprosthetic hip and knee infection: an evidence-based and validated criteria. J Arthroplasty. 2018, 33:1309-14. 10.1016/j.arth.2018.02.078

10. Manchanda V, Sanchaita S, Singh N: Multidrug resistant acinetobacter. J Glob Infect Dis. 2010, 2:291-304. 10.4103/0974-777X.68538

11. De Silva RND, Jayatilleke SK, Perera A: Successful treatment of prosthetic joint infection with multidrug resistant Acinetobacter species with debridement and retention: a case report. Sri Lankan J Inf Dis. 2016, 6:55-8. 10.4038/sljid.v6i1.8104

12. Donskey CJ: Does improving surface cleaning and disinfection reduce health care-associated infections? Am J Infect Control. 2013, 41:S12-9. 10.1016/j.ajic.2012.12.010

13. Uhel F, Corvaisier G, Poinsignon Y, et al.: Mycobacterium tuberculosis prosthetic joint infections: a case series and literature review. J Infect. 2019, 78:27-34. 10.1016/j.jinf.2018.08.008

14. Martínez-Pastor JC, Muñoz-Mahamud E, Vilchez F, et al.: Outcome of acute prosthetic joint infections due to gram-negative bacilli treated with open debridement and retention of the prosthesis. Antimicrob Agents Chemother. 2009, 53:4772-7. 10.1128/AAC.00188-09

15. Kazimoglu C, Yalcin N, Onvural B, Akcay S, Agus H: Debridement, antibiotics, irrigation, and retention (DAIR) of the prosthesis after hip hemiarthroplasty infections. Does it work?. Int J Artif Organs. 2015, 38:454-60. 10.5301/ijao.5000430

16. Sukeik M, Patel S, Haddad FS: Aggressive early débridement for treatment of acutely infected cemented total hip arthroplasty. Clin Orthop Relat Res. 2012, 470:3164-70. 10.1007/s11999-012-2500-7

17. Aboltins CA, Dowsey MM, Buising KL, Peel TN, Daffy JR, Choong PF, Stanley PA: Gram-negative prosthetic joint infection treated with debridement, prosthesis retention and antibiotic regimens including a fluoroquinolone. Clin Microbiol Infect. 2011, 17:862-7. 10.1111/j.1469-0691.2010.03361.x

18. Jaén N, Martínez-Pastor JC, Muñoz-Mahamud E, García-Ramiro S, Bosch J, Mensa J, Soriano A: Long-term 


\section{Cureus}

outcome of acute prosthetic joint infections due to gram-negative bacilli treated with retention of prosthesis. Rev Esp Quimioter. 2012, 25:194-8.

19. Vila A, Pagella H, Amadio C, Leiva A: Acinetobacter prosthetic joint infection treated with debridement and high-dose tigecycline. Infect Chemother. 2016, 48:324-9. 10.3947/ic.2016.48.4.324

20. Corvec S, Furustrand Tafin U, Betrisey B, Borens O, Trampuz A: Activities of fosfomycin, tigecycline, colistin, and gentamicin against extended-spectrum- $\beta$-lactamase-producing Escherichia coli in a foreignbody infection model. Antimicrob Agents Chemother. 2013, 57:1421-7. 10.1128/AAC.01718-12 